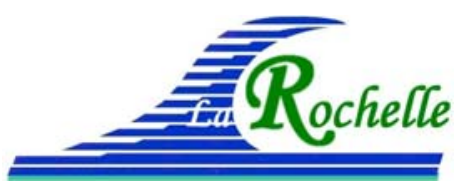

XVèmes Journées Nationales Génie Côtier - Génie Civil La Rochelle, 29 au 31 mai 2018

DOI:10.5150/jngcgc.2018.100 ～(C) Editions Paralia CFL

disponible en ligne - http://www.paralia.fr - available online

\title{
Modulariser les formations universitaires en génie côtier pour faciliter l'accès des professionnels : le programme UN e-SEA
}

\section{Noémie LARROUILH ${ }^{1}$, Noémie FOUCHER ${ }^{1}$, Gwenaele PROUTIERE-MAULION ${ }^{1,2}$, Franck SCHOEFS ${ }^{3}$, Martin SANCHEZ ${ }^{4}$, Emilio BASTIDAS-ARTEAGA ${ }^{3}$, Jérôme LEBEAU ${ }^{5}$}

1. Université de Nantes, UN e-SEA, 2 rue de la Houssinière, 44322 Nantes, France. noemie.larrouilh@univ-nantes.fr ; noemie.foucher@univ-nantes.fr

2. Université de Nantes, Présidence, EA 1165 CDMO, IUML FR 3473 CNRS, Direction des relations internationales, 1 quai de Tourville, BP 13522, 44035 Nantes, France.

3. Université de Nantes, UFR Sciences et Techniques, GeM UMR 6183 CNRS / IUML FR 3473 CNRS, 2 rue de la Houssinière, 44322 Nantes, France.

4. Université de Nantes, UMR-6112 CNRS - Planétologie et Géodynamique, 2 rue de la Houssinière, 44322 Nantes cedex 3, France.

5. Herskovits \& Tobie Architecture navale et Ingénierie maritime, 9 rue Jeanne d'Arc, 44000 Nantes, France.

\section{Résumé :}

A travers le programme UN e-SEA, l'université de Nantes développe une version à distance du master de Travaux Publics, Maritimes et Maintenance, adaptée aux professionnels du génie civil afin de leur en faciliter l'accès, et ce partout dans le monde, sans que cela ne nécessite une interruption de l'activité professionnelle.

La démarche de modularisation permet de prendre en compte la diversité des bénéficiaires (métier, cursus préalable, lieu d'exercice, ...), tout en adoptant des formats propices à la formation continue.

\section{Mots-clés :}

Formation professionnelle, Enseignement à distance, Approche par compétences, Génie civil, Hydraulique maritime, Méthodes de réalisation des ouvrages, Constructions offshore, Aménagement portuaire, Maintenance, Auscultation, Génie mécanique, Génie Côtier.

\section{Introduction}

Face à la maritimisation croissante des économies, les enjeux de l'aménagement côtier et offshore se multiplient. Il en résulte des besoins en formation variés, y compris pour les professionnels du génie civil déjà en activité.

Les formations universitaires, telles que, à l'université de Nantes, le Master de Génie Civil, Parcours Travaux Publics, Maritimes et Maintenance (TP2M ; UNIVERSITE DE NANTES, 2017a ; 2017b \& 2017c), sont particulièrement aptes à traiter, d'une part, la pluralité des enjeux du génie côtier, et d'autre part, la réactualisation scientifique 


\section{Hors session}

continue des compétences. Cependant, conçues pour répondre aux prérogatives d'un public étudiant, elles nécessitent d'être profondément réaménagées, afin de répondre aux attentes des professionnels du secteur économique et de la recherche.

A travers le programme UN e-SEA (site web), l'université de Nantes a fait le choix de la digitalisation des formations, ceci afin d'opérer les aménagements nécessaires à la prise en charge des publics professionnels partout dans le monde.

\section{Le programme UN e-SEA}

UN e-SEA, université numérique en sciences de la mer, est un programme mis en place par l'université de Nantes, avec le soutien de la Région des Pays de la Loire et de Nantes Métropole, afin de faciliter l'accès des professionnels et futurs professionnels, à des formations de niveau Master en sciences de la mer. En faisant largement appel, mais pas exclusivement, aux technologies d'enseignement à distance, UN e-SEA permet :

- L'accès à la formation aux publics n'ayant pas le loisir de se déplacer régulièrement pour se former (professionnels, navigants, personnes résidant à l'étranger, ...),

- La modularisation des formations selon les besoins (rythme, format, adaptation des contenus à un contexte local donné).

\section{Modularisation des formations UN e-SEA en génie côtier}

La transformation du master Travaux Publics Maritimes et Maintenance (TP2M) en formation e-learning s'opère selon 5 principes développés dans ce qui suit.

\subsection{S'appuyer sur une approche par compétences (GHAZEL, 2012)}

Objectifs : Etablir une cohérence entre les objectifs pédagogiques, les contenus de formation, les activités d'entraînement et leur mise en pratique en contexte professionnel.

Démarche: Construire un scénario de formation global, à partir d'objectifs pédagogiques clairement structurés en fonction d'un référentiel métier et d'une taxonomie didactique appropriée.

Finalité : Grille d'objectifs, susceptible d'être réadaptée en fonction de besoins spécifiques locaux. 


\section{XVèmes Journées Nationales Génie Côtier - Génie Civil \\ La Rochelle, 29 au 31 mai 2018}

\subsection{Réviser le séquençage des formations}

Objectifs : Adapter le séquençage au caractère extensif de la formation. Adapter le séquençage au caractère "à la carte" de la formation.

Démarche: Les enseignements spécifiquement maritimistes sont extraits du programme de master et constituent le cour du travail de modularisation. Les autres enseignements sont validés par équivalence universitaire, ou validation des acquis de l'expérience professionnelle.

Les règles de séquençage s'appuient sur le résultat d'études cognitivistes sur l'apprentissage (temps d'attention, surcharge cognitive, ...).

Les grains pédagogiques sont agencés de manière à faciliter les interventions sur les contenus.

Les passerelles de progression sont à triple entrée : par niveau, par thématique, par durée des formations.

Finalité : 18 formations courtes (12 à 22 heures) combinées en 4 formations certifiantes (60 à 90 heures chacune): Méthodes de réalisation des ouvrages maritimes ; Aménagement portuaire ; Auscultation, monitoring et maintenance des constructions offshore et côtières; Risques et fiabilité des constructions offshore et côtières.

L'accomplissement cumulé de ces 4 certificats, associé à la validation des acquis de l'expérience et à la production d'un écrit défendu à l'occasion d'une soutenance (mémoire, rapport d'activité, ...), peut donner lieu, en cas de succès, à la délivrance du Master de Travaux Publics Maritimes et Maintenance de l'université de Nantes.

3.3 Former les formateurs aux spécificités de l'enseignement à distance

Objectifs: Permettre aux formateurs de s'approprier le cahier des charges pédagogique du programme UN e-SEA.

Maintenir, dans un contexte distanciel, une qualité de formation au moins égale à celle du master en présentiel.

Responsabiliser le formateur vis-à-vis du cours produit et des personnes formées.

Démarche: Rendez-vous individuel de sensibilisation aux spécificités de l'enseignement à distance avec chaque formateur impliqué (Décret $\mathrm{n}^{\circ}$ 2014-935 du 20 aout 2014).

Accompagnement continu pendant la production du cours.

Le cas échéant, formations complémentaires à certaines techniques pédagogiques spécifiques mises en œuvre.

Finalité : $\quad$ Fiches repères à l'usage des formateurs.

Carte mentale du cours (pour chaque cours, un modèle pédagogique spécifique défini par le formateur et l'ingénieur pédagogique). 


\section{Hors session}

3.4 Adapter les modalités de formation (formats, medias, activités, évaluations)

Objectifs: Optimiser la progression par le choix des médias et activités pédagogiques.

Démarche: Le choix des médias et des activités pédagogiques résulte du meilleur compromis entre le profil du formateur, le profil des bénéficiaires, l'objectif pédagogique, le type d'activités pratiques à mettre en place, l'ergonomie d'utilisation.

Finalité : $\quad$ Syllabus - Espace d'accueil - Guidage textuel - Tutoriels - AutoEvaluation - Tutorat.

3.5 Mettre en œuvre un système de reconnaissance scientifique, professionnelle et universitaire

Objectifs : $\quad$ Permettre au stagiaire de valoriser la formation souhaitée quel que soit le but recherché.

Démarche : Circuits de reconnaissance interne VAE.

Certification FCU (BUREAU VERITAS CERTIFICATION, 2016).

Eligibilité au CPF (en cours).

Finalité : Financement, progression de carrière, reconversion professionnelle, référencement des compétences, doctorat.

\section{Conclusion}

Globalement, la démarche de transformation du master vers la formation professionnelle s'appuie sur les fondamentaux de la pédagogie et de la gestion de projet: analyse du besoin et des objectifs, analyse des prérequis, progression vers des objectifs pédagogiques ciblés.

L'originalité et la qualité des contenus telles qu'elles existent dans la formation en présentiel, sont maintenues : approche par projet et études situationnelles, actualisation permanente des contenus, prise en compte des enjeux juridiques, socio-économiques, anthropiques, de l'aménagement du littoral et de l'espace maritime.

La modularisation par les techniques d'enseignement à distance permet une réelle plusvalue pour les professionnels, à condition d'en maîtriser les impacts par un cadrage pédagogique rigoureux.

Une attention particulière est à porter sur les dispositifs d'auto-évaluation des formations dispensées, dans l'objectif de garantir leur efficacité et leur amélioration continue.

Pistes de développement : exploiter la diversité des profils des stagiaires, et le caractère international des promotions constituées, dans le cadre d'une mise en réseau des stagiaires et des partenaires internationaux susceptibles de participer à la production de contenus de formation. 


\section{XVèmes Journées Nationales Génie Côtier - Génie Civil \\ La Rochelle, 29 au 31 mai 2018}

\section{Références}

BUREAU VERITAS CERTIFICATION (2016). La Formation Continue à l'Université

- La Force d'un réseau Public: Référentiel de certification de services (Ref: RE/FCU/1,6 version 2.1 du 16/12/2016).

GHAZEL T. (2012). L'approche par compétences : définition et principes (article web). URL : http://tarekghazel.ek.la/l-approche-par-competence-definition-et-principes-a29373531

JORF $n^{\circ} 0193$ du 22 août 2014. Décret $n^{\circ}$ 2014-935 du 20 aout 2014 relatif aux formations ouvertes ou à distance (page 13958, texte $n^{\circ} 30$ ).

UN e-SEA -Université numérique des sciences de la mer- (site web). http://www.unesea.univ-nantes.fr/

UNIVERSITE DE NANTES (2017a). Master 1 M1 Travaux Publics, Maritimes et Maintenance, années universitaires 2017-2021 (accréditation quinquennale).

UNIVERSITE DE NANTES (2017b). Master 2 M2 Travaux Publics et Maritimes, années universitaires 2017-2021 (accréditation quinquennale).

UNIVERSITE DE NANTES (2017c). Master 2 M2 Travaux Publics et Maintenance, années universitaires 2017-2021 (accréditation quinquennale). 
Hors session 